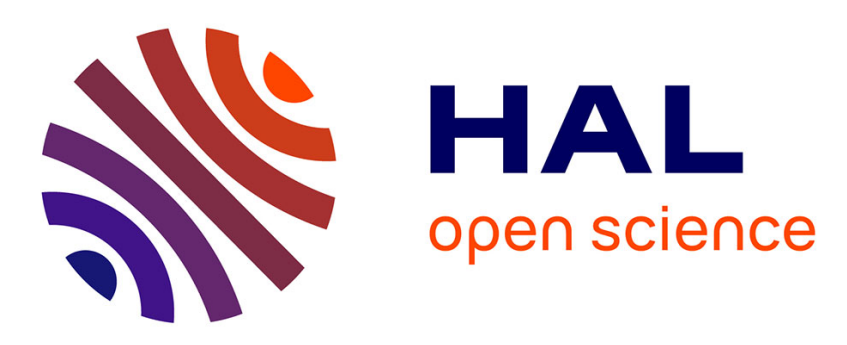

\title{
Nitric oxide participates at the first steps of Apis mellifera cellular immune activation in response to non-self recognition
}

Pedro Negri, Matías Maggi, Natalia Correa-Aragunde, Constanza Brasesco, Martín Eguaras, Lorenzo Lamattina

\section{To cite this version:}

Pedro Negri, Matías Maggi, Natalia Correa-Aragunde, Constanza Brasesco, Martín Eguaras, et al.. Nitric oxide participates at the first steps of Apis mellifera cellular immune activation in response to non-self recognition. Apidologie, 2013, 44 (5), pp.575-585. 10.1007/s13592-013-0207-8 . hal01201327

\section{HAL Id: hal-01201327 \\ https://hal.science/hal-01201327}

Submitted on 17 Sep 2015

HAL is a multi-disciplinary open access archive for the deposit and dissemination of scientific research documents, whether they are published or not. The documents may come from teaching and research institutions in France or abroad, or from public or private research centers.
L'archive ouverte pluridisciplinaire HAL, est destinée au dépôt et à la diffusion de documents scientifiques de niveau recherche, publiés ou non, émanant des établissements d'enseignement et de recherche français ou étrangers, des laboratoires publics ou privés. 


\title{
Nitric oxide participates at the first steps of Apis mellifera cellular immune activation in response to non-self recognition
}

\author{
Pedro Negri ${ }^{1,3,4}$, Matías MagGi ${ }^{1,3}$, Natalia Correa-Aragunde ${ }^{2,3}$, \\ Constanza Brasesco ${ }^{1}$, Martín Eguaras ${ }^{1,3}$, Lorenzo Lamattina ${ }^{2,3}$

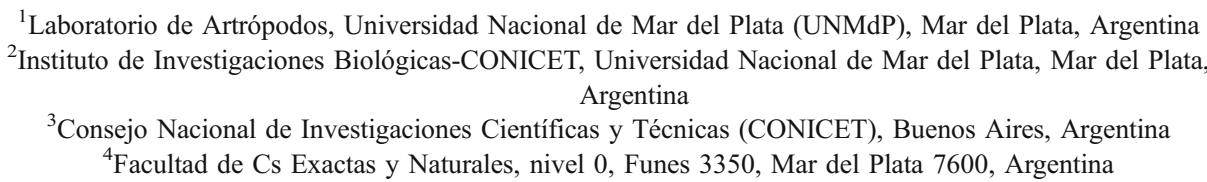

Received 19 November 2012 - Revised 18 March 2013 - Accepted 27 March 2013

\begin{abstract}
The honey bee Apis mellifera (Hymenoptera) is being affected by many diseases. The elimination of organisms in the insect hemocoel requires hemocytes recognition and response to the invader. After recognizing a surface as "foreign," hemocytes "spread." Spreading on glass surfaces by insect hemocytes is used as a measure of immune activation. Nitric oxide (NO) is a signaling and immune effector molecule in response to microbial infection that has been proposed as a key molecule in invertebrate immunity. The participation of $\mathrm{NO}$ in the hemocytic response of A. mellifera upon recognition of non-self is herein analyzed. Glass-adherent hemocytes produce large amounts of NO. Treatment with NO donor sodium nitroprusside enhanced hemocyte spreading, while NO scavenger carboxyPTIO reduced hemocyte immune activation. These results are indicative of NO participation at the beginning of $A$. mellifera immune response to non-self.
\end{abstract}

\section{Apis mellifera / immunity / nitric oxide}

\section{INTRODUCTION}

The honey bee Apis mellifera is known to be affected by many parasites that play a key part in the insect development, and pose a serious threat to the ecosystem, agriculture, and apiculture activities. Among parasites affecting bee health, one of the main pests affecting bee colonies is the parasitic mite Varroa destructor which has been postulated to be one of the worldwide colonies responsible for colony losses reported as the

M. Eguaras and L. Lamattina contributed equally as senior authors.

Corresponding author: P. Negri, pedronegri1@yahoo.com.ar Manuscript editor: Klaus Hartfelder colony collapse disorder (CCD) syndrome (Martin et al. 2012; Dietemann et al. 2012; Levy 2012; Rosenkranz et al. 2010; Potts et al. 2010). Another serious threat that harms A. mellifera is American foulbrood (severe bacterial disease affecting bee larvae) caused by Paenibacillus larvae, and the emergent microparasite Nosema ceranae (internal fungi affecting bee gut). Lately, it has been proposed that the combined effects of all these parasites and/or pathogens with habitat loss and pesticide poisoning drastically affect honey bees resulting in CCD (Martin et al. 2012; Dietemann et al. 2012; Levy 2012; Hendriksma et al. 2011). In this sense, the study of $A$. mellifera immune system could provide with basic information to better understand the way in which honey bees defend themselves against parasites. 
Insects defend themselves against infectious organisms by a well-developed innate immune system (Strand 2008). The elimination of organisms that enter the insect hemocoel requires blood cells (hemocytes) able to recognize and respond to the invading species (Clark et al. 1997; Marmaras and Lampropoulou 2009). Upon recognizing a surface as foreign, hemocytes quickly transform from resting non-adherent cells into activated adherent cells that first adhere as a monolayer or as foci to the foreign surface (Nardi et al. 2006). After "non-self" recognition, hemocytes respond by "spreading" (Gillespie and Kanost 1997). If the foreign agent is small, this spreading ability promotes particle phagocytosis. When it is larger (or many small foreign objects together), nodulation or encapsulation occurs due to the cooperative action of a number of hemocytes (Eleftherianos et al. 2009). During encapsulation, hemocytes adhere to the surface of non-self particles and to each other, and spread forming layers of overlapping cells which then destroy the particle by secreting cytotoxic compounds (Whitten et al. 2004). Indeed inhibiting hemocyte spreading is a common strategy employed by many entomopathogens; and such changes in hemocyte behavior are important components of insect defense (Eleftherianos et al. 2009). Many endogenous signals, thought to be released by hemocytes or wounded tissue, also influence hemocyte spreading (Strand 2008; Eleftherianos et al. 2009).

Biotic microbial and non-microbial surfaces as well as abiotic surfaces such as plastic and glass are recognized as non-self by the insect innate immune system and encapsulated by hemocytes. Consistently, spreading on a glass surface by insect hemocytes is often used as a measure of immune fitness (Dean et al. 2004).

Nitric oxide (NO) is a highly reactive multifunctional free radical generated during the oxidation of L-arginine to L-citrulline by the enzyme NO synthase (NOS). A wide range of physiological functions controlled by NO signaling have been described as governed by the activation of different NOS isoforms and by the rapid diffusion of this molecule across cell membranes Davies and Dow (2009). In vertebrates, three main types of this enzyme have been found: two constitutive NOS (cNOS), endothelial NOS, and neuronal NOS, respectively; and one inducible NOS (iNOS) (Rivero 2006). In invertebrates, just one NOS isoform has been detected with either a constitutive (signaling) or an inducible (toxic) role. In some cases, the same isoform has been found to have both roles (Rivero 2006). Numerous reviews have described central roles for NO signaling in host defense mechanisms against infections caused by viruses, bacteria, protozoan, and metazoan parasites. In addition, NO acts as a non-specific cytotoxic molecule (Rivero 2006). Cellular toxicity results from the local oxidation of large amounts of NO into peroxynitrite and other unstable molecules with DNA-, protein-, and lipid-damaging properties (Hillyer and Estévez-Lao 2010). In insects, NO is produced as an immune effector molecule in response to microbial infection in several species of lepidopterans, hemipterans, and dipterans (Rivero 2006). Therefore, NO has been proposed as a key molecule to confront parasites in invertebrates (Rivero 2006).

Regarding NO signaling in honeybees, most works have centered on the role that NO plays as a neurotransmitter in learning (Müller and Hildebrandt 1995; Müller 1996; Müller and Hildebrandt 2002). So far, there is scant information available about NO participation in $A$. mellifera immune defense, and the only report in this regard is the one by Negri et al (2012a).

This work aims to study the role of NO in $A$. mellifera cellular immunity. In particular, it focuses on the part that NO has in A. mellifera hemocyte immune activation after non-self recognition.

\section{MATERIALS AND METHODS}

\subsection{Insects}

A. mellifera brood combs were collected from the J. J. Nagera experimental apiary of the Arthropods Laboratory of the Universidad Nacional de Mar del Plata located on Ruta no. 11 ( $\left.38^{\circ} 10^{\prime} 06^{\prime \prime} \mathrm{S} ; 57^{\circ} 38^{\prime} 10^{\prime \prime} \mathrm{W}\right)$. Combs were cleaned off from workers and taken into an incubator. Two developmental bee stages were used in the experiments: newly emerged workers (W) that emerged in the incubator at $30-32{ }^{\circ} \mathrm{C}$ and $70 \%$ relative humidity, and fifth instar larvae (L5). 


\subsection{Hemolymph collection}

Hemolymph collection was performed as previously described by Negri et al (2012b). In short, insect blood was collected from fifth instar larvae by puncturing the soft cuticle with a sterile $30-\mathrm{G}$ needle. Workers were punctured in the neck at the aorta and hemolymph was collected from the resulting bubble. Modified plastic tubes were used to immobilize the adult honey bee and expose the aorta. Before puncturing, the cuticle was surface sterilized with $70 \%$ ethanol. The hemolymph was collected with a micropipette and transferred into a microcentrifuge tube containing ice-cold sterile anticoagulant buffer (AB) $(0.098 \mathrm{M} \mathrm{NaOH}, 0.186 \mathrm{M} \mathrm{NaCl}$, 0.017 M EDTA, and 0.041 M citric acid, pH 4.5) (Pech and Strand 1995), phosphate-buffered saline (PBS, $\mathrm{pH}=7.2$ ) or culture media. Three culture media were used: Grace's Insect Medium (GIM, Hyclone), TC-100 insect cell culture medium (Sigma), or Excell-405 culture medium (Sigma) (1:10 ratio of hemolymph/ PBS medium). Hemolymph solution was homogenized by soft pipetting into the sampling tube and then transferred to 96-sterile-well glass-bottom plates (NUNC, 96-well optical bottom plates, sterile) where all the experiments were performed. In experiments, where the non-adherent-to-glass hemocytes were separated from the adherent ones, the cells were resuspended into $\mathrm{AB}$ to avoid cell clumping. Twenty minutes after plating the cells, the non-adherent hemocytes were removed using a micropipette and transferred to another well. The remaining adherent hemocytes were again covered with buffer and the non-adherent-to-glass ones deposited in another well for further observations, and maintained within the same buffer.

\subsection{Nodulation assay}

The methods described by Pech and Strand (1995) and Ling and $\mathrm{Yu}$ (2005) were combined and modified resulting in the methodology described by Negri et al (2012b). Briefly, in order to avoid hemocyte adherence, the glass surface of the bottom of the plates was covered with $30 \mu \mathrm{L}$ of $1 \%$ agarose. This resulted in a thin layer of agarose that allowed keeping hemocytes in suspension and visualize them in real time. Many different time points could be performed with no need of many fixation and washing steps. To stimulate nodulation, a $10-\mu \mathrm{L}$ micropipette tip was first introduced into an Escherichia coli suspension and then used to touch the center of the agarose thin layer before adding the hemocyte suspension. Bacteria were washed into the culture media used to sample the hemolymph in each experiment before bacterial challenge. Hemocytes cultured in agarosecoated wells without seeded bacteria were used as the control groups. This methodology allowed to observe hemocytes responding to the challenge as well as those not responding to bacterial nodulation on a simultaneous basis, within the same well where $E$. coli was seeded. This represents a better resolution for the analysis of NO production within hemocytes in relation with the nodulation response.

\subsection{Nitric oxide detection and quantification}

The endogenous synthesis of NO in living hemocytes was evidenced by means of the fluorescent probe DAF-FM-DA (Invitrogen) at a final concentration of $5 \mu \mathrm{M}$. This dye emits green fluorescence within cells after reacting with NO. Two methodologies were followed to measure NO production within hemocytes: Fluorometry (Fluoroskan Ascent, Labsystems, excitation at $480 \mathrm{~nm}$-emission at $520 \mathrm{~nm}$ ) for NO production measurements over time (up to $6 \mathrm{~h}$ ), and green fluorescence quantification from the pictures taken in the microscope after fluorometer measurements using ImageJ (National Institute of Health, USA, public domain software). This procedure enhanced measurements quality as it allowed to confirm that the fluorescence detected by the fluorometer was emitted by the hemocytes.

\subsection{Hemocyte spreading assays}

Cells spreading upon stimulation with the glass surface were considered immune-related hemocytes. Half of the volume from each hemolymph containing solution was deposited on an equal volume of PBS/ media alone or PBS/media containing each treatment. The same hemolymph sample was equally distributed in each treatment so as to conduct the experiments under standardized conditions regarding hemocytes number and cells "life history." Avoiding hemocyte count before the experiments reduced the manipulation of the hemocytes to be analyzed in the assays. Hemocytes were collected and cultured as described above and the 
media was supplemented with the NO donor sodium nitroprusside (SNP, Sigma) or with the endogenous NO scavenger carboxy-PTIO (CPTIO, Invitrogen). The same hemolymph sample was equally distributed in each treatment. The cellular spreading of the first adherent-to-glass hemocyte type that spread after glass contact was measured using ImageJ. The effects of CPTIO treatment was evaluated over time for up to $24 \mathrm{~h}$ following the same procedure.

\subsection{Microscopy}

After being transferred to the 96-well plates, the mixtures containing A. mellifera hemolymph were observed for $24 \mathrm{~h}$ using inverted microscopy. Hemocytes were examined by means of differential interference contrast or epifluorescence (excitation at $480 \mathrm{~nm}$-emission at $520 \mathrm{~nm}$ ) microscopy. The microscope used was a Nikon Eclipse $T i$ inverted microscope using a $\times 60$ objective.

\subsection{Statistics}

To evaluate NO production in different hemocyte types a Mann-Whitney test was performed (Figure 3), while a $T$ test was applied (Figure 4) to analyze the effects of SNP and CPTIO on hemocyte spreading. The effects of CPTIO in time course experiments was evaluated by means of a two-way ANOVA test followed by multiple comparisons (Figures 5 and 6) applying a Bonferroni correction (alpha equal to 0.01; Bonferroni correction was only applied to data in Figure 5).

\section{RESULTS}

\subsection{Nitric oxide is produced after non-self recognition in $A$. mellifera hemocytes}

To assess the endogenous NO production after non-self recognition, A. mellifera worker hemocytes were challenged with abiotic and biotic non-self. A. mellifera hemocytes were challenged directly with glass to trigger abiotic non-self recognition. Attachment to glass is considered as an immune response to abiotic non-self recognition in insect hemocytes. The biotic challenge reported to induce NO production within innate-immunity-related cells is E. coli.
Therefore, an in vitro nodulation assay was carried out to study the correlation between endogenous NO production by $A$. mellifera hemocytes and biotic non-self recognition. The endogenous NO production of $A$. mellifera hemocytes was evaluated in vitro using the specific fluorescent dye for NO detection DAF-FM-DA. The green fluorescence within the cells was indicative of endogenous NO production. When $A$. mellifera hemocytes where challenged directly with glass, NO production was detected particularly within the adherent-to-glass hemocyte type (Figure 1). In the nodulation assays, A. mellifera hemocytes agglomerated over $E$. coli bacteria resulting in a multicellular response. The hemocytes agglomerated over the bacteria featured a circular shape in agreement with the tip shape used to seed the bacterial challenge. Interestingly, the green fluorescence associated to $\mathrm{NO}$ production was observed in strict relation with hemocytes agglomeration (Figure 2). The hemocytes within the same well that were neither implicated in the nodulation response nor able to contact the glass bottom (because this was covered with $1 \%$ agarose) did not emit green fluorescence.

\subsection{A. mellifera adherent-to-glass hemocytes produce more nitric oxide than non-adherent-to-glass ones do}

To investigate the relationship between $A$. mellifera worker hemocyte types and NO production after abiotic non-self recognition even further, experiments in which hemocytes adherent-to-glass were distinguished from those non-adherent-to-glass types were performed. This resulted in wells containing adherent-toglass hemocytes and wells with non-adherentto-glass cells (Figure 3a). NO production was detected by the fluorescent probe DAF-FM-DA. Green fluorescence was measured for up to $6 \mathrm{~h}$ after glass challenge. Adherent-to-glass hemocytes showed increased NO production in time after glass attachment (Figure 3b). Moreover, they showed much more fluorescence associated to NO production than non-adherent ones did after the time studied (Figure 3c). 


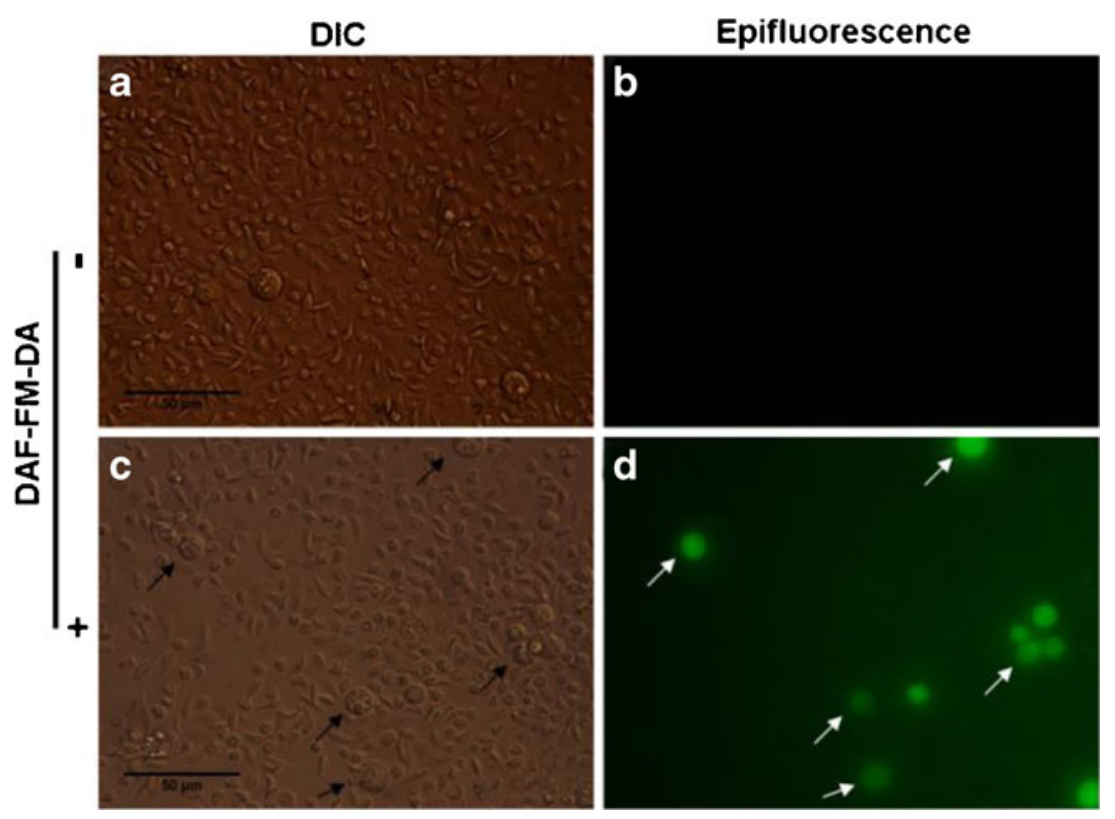

Figure 1. A. mellifera worker hemocytes produce nitric oxide (NO) after glass attachment. A. mellifera hemocytes were cultured in vitro in 96-well glass-bottom plates. The hemocytes that were not loaded with the fluorescent dye DAF-FM-DA were used as self-fluorescence control $(\mathbf{a}, \mathbf{b})$. NO production was evaluated using $5 \mu \mathrm{M}$ DAF-FM-DA (c, d). DAF-FM-DA emits green fluorescence upon reaction with NO within living cells. Arrows indicate adherent-to-glass hemocytes. Scale bar $=50 \mu \mathrm{m}$.

\subsection{Nitric oxide participates in hemocyte spreading after non-self recognition}

A series of experiments were conducted to study the effect of NO on $A$. mellifera worker hemocytes spreading upon non-self recognition by treating the hemocytes with the $\mathrm{NO}$ donor SNP and the endogenous NO scavenger CPTIO. The hemocyte type was selected for being the first $A$. mellifera hemocytes that adhered to
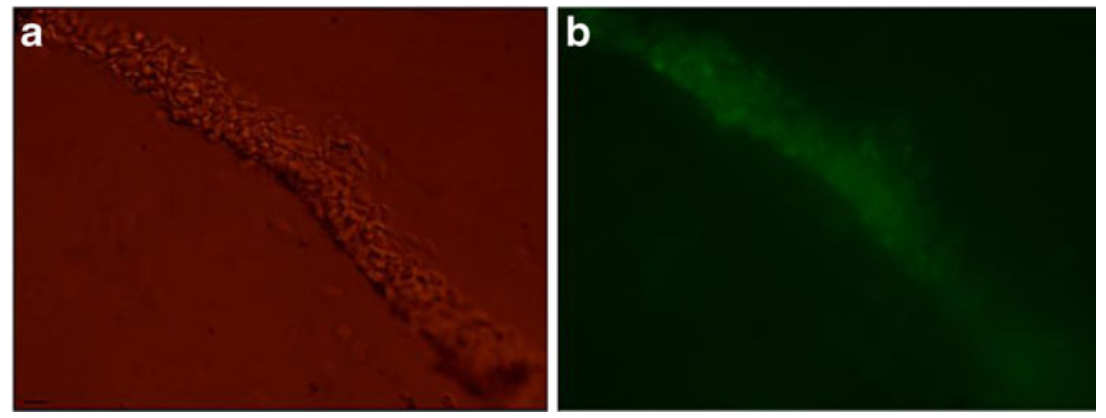

Figure 2. E. coli triggers $A$. mellifera worker hemocytes agglomeration and nitric oxide (NO) production. $A$. mellifera hemocytes were cultured in agarose-coated-bottom wells with seeded bacteria (E. coli) on the agarose layer. Hemocytes were loaded with the fluorescent probe DAF-FM-DA $(5 \mu \mathrm{M})$ which produces green fluorescence upon reaction with NO within cells. a Agglomeration of the hemocytes co-localize with bacteria location. b NO production correlates with hemocytic agglomeration. Scale bar $=10 \mu \mathrm{m}$. 

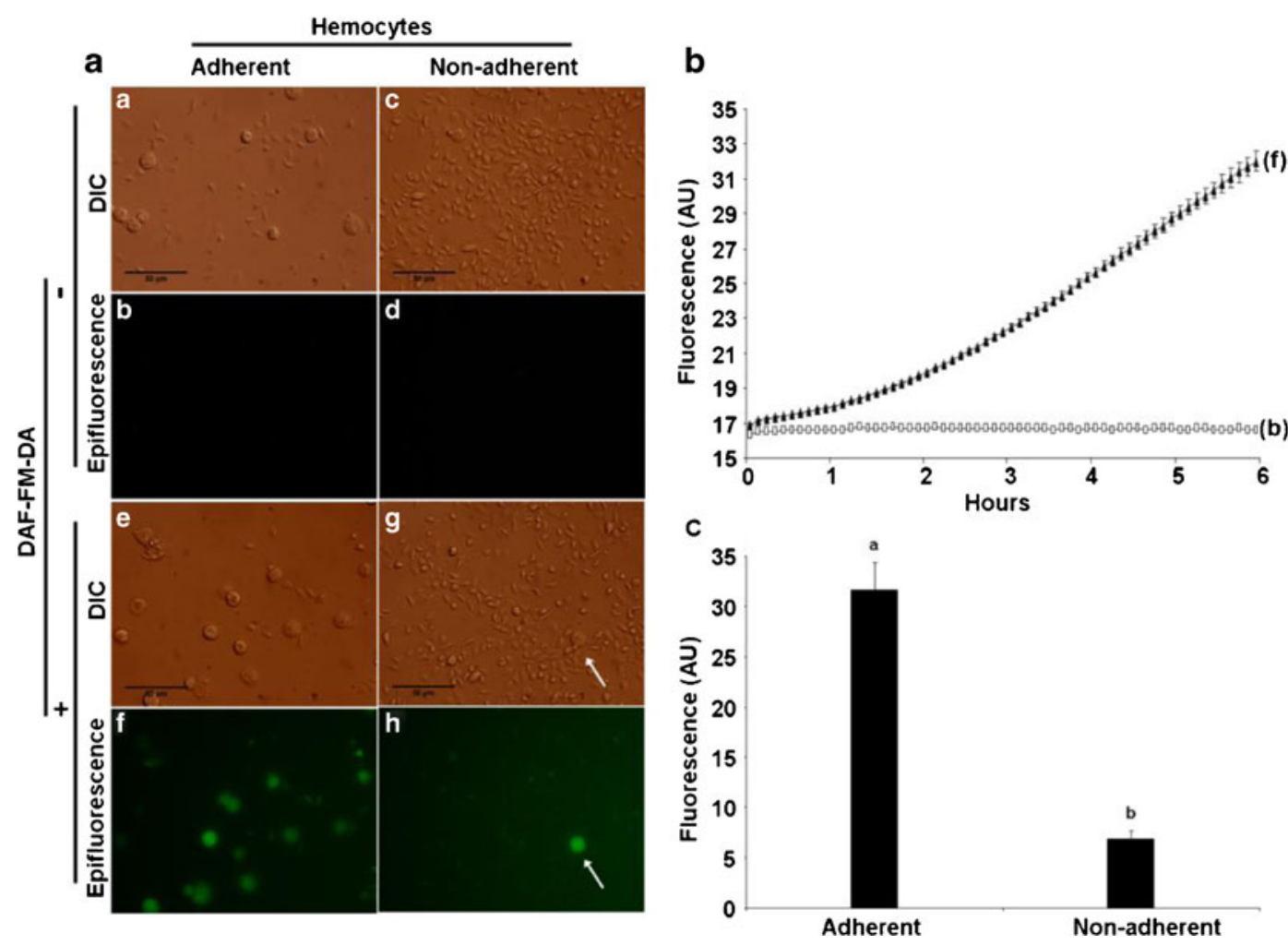

Figure 3. Adherent-to-glass A. mellifera worker hemocytes produce more nitric oxide (NO) than non-adherentto-glass do. a Adherent-to-glass hemocytes (adherent; a, e) were separated from non-adherent-to-glass ones (non-adherent; c, g) after 20 min of culture in 96-well glass-bottom plates. The non-adherent hemocytes were placed in another well. Then, the NO production was analyzed by means of the DAF-FM-DA fluorescent probe at a concentration of $5 \mu \mathrm{M}$. DAF-FM-DA emits green fluorescence upon reaction with NO within cells (f, $\mathbf{h})$. The hemocytes that were not loaded with the fluorescent dye were used as self-fluorescence control (b, d). b The green fluorescence of the adherent hemocytes was measured for up to $6 \mathrm{~h}$ using a fluorometer. $\mathbf{c}$ The fluorescence after $6 \mathrm{~h}$ emitted by adherent and non-adherent hemocytes was quantified from the pictures using ImageJ. Different letters mean statistical differences in NO production between each hemocyte type. MannWhitney, $P=<0.001 ; n=30$. $A U$ means "arbitrary units." The white arrow points to an adherent-to-glass hemocyte type contaminating the non-adherent-to-glass hemocytes well. Scale bar $=50 \mu \mathrm{m}$.

glass in vitro (referred to as adherent-to-glass hemocytes). Cellular spreading over glass was significantly enhanced with the SNP treatment. Conversely, it was significantly diminished with respect to control when treated with CPTIO (Figure 4).

Since A. mellifera hemocyte spreading is a dynamic process, experiments were performed to assess the effect of endogenous NO scavenging on hemocytes spreading over time. These experiments were carried out using hemocytes obtained from newly emerged workers (Figure 5) and L5 larvae (Figure 6). The hemocyte type under study was selected for being the first $A$. mellifera hemocyte type to adhere to glass in vitro in each stage under study (Negri et al. 2012b). In workers, hemocytes spreading was significantly reduced by CPTIO treatments as compared to the control group (Figure 5; two-way ANOVA, $f=8.78, P=0.006$ ). However, as illustrated in Figure 5, the differences between the two CPTIO concentrations used were not significant 


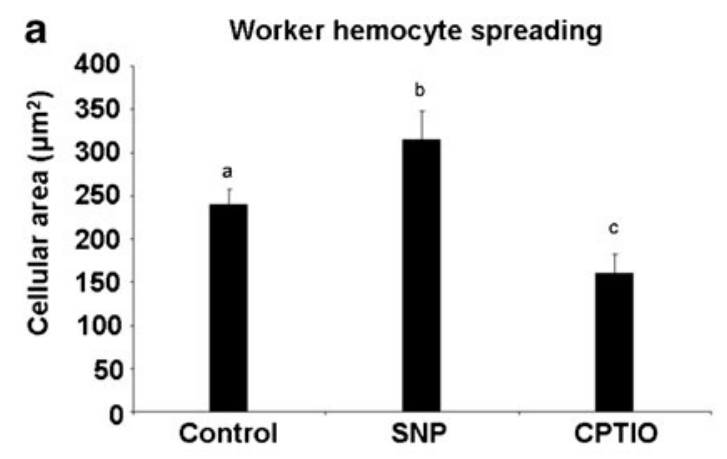

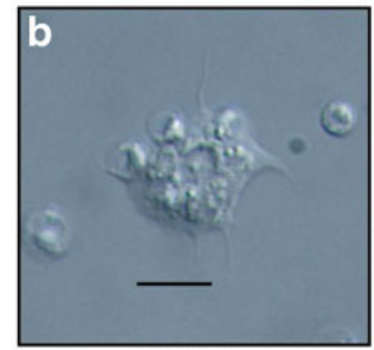

Control

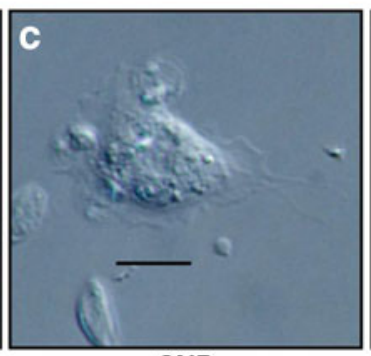

SNP

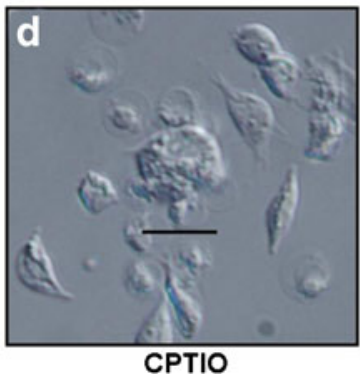

Figure 4. Nitric oxide (NO) participates in A. mellifera hemocytes spreading upon non-self recognition of glass surfaces. A. mellifera worker hemocytes were cultured in 96-well glass-bottom plates. The fact that adherent-toglass hemocytes spread evidences immune activation, and it was measured as cellular area using ImageJ software (a). Different letters mean statistical differences in hemocyte spreading compared to control; $a$ vs. $b, T$ test $P=0.036$; $a$ vs. $c, T$ test $P=0.018 ; n=15$. Hemocytes cultured in media alone (control, b) or media plus the exogenous NO donor SNP (SNP, $0.1 \mathrm{mM}$, c) or media supplemented with the NO scavenger CPTIO (CPTIO, $0.1 \mathrm{mM}, \mathbf{d})$. Scale bars $=10 \mu \mathrm{m}$.

$(P>0.01)$. Yet a significant effect in hemocyte spreading was detected through time for each treatment (two-way ANOVA, $f=4.98 ; P=0.01$ ): T0 was statistically different at 90, 170, and $230 \min (P<0.01)$. Nitric oxide scavenging through CPTIO treatment also reduced spreading on a significant basis in L5 hemocytes (Figure 6; two-way ANOVA $f=24.65 ; P=0.01$ ). However, no significant effect in hemocyte spreading was detected in L5 hemocytes (two-way ANOVA, $f=6.96 ; P=0.07)$ through time. Interestingly, CPTIO-treated hemocytes copied the spreading curve described by the control cells, though the response studied was always lower for the two stages analyzed in the CPTIO-treated hemocytes (Figures 5 and 6). This indicates that $\mathrm{NO}$ is required for the correct spreading of the hemocytes obtained from workers and L5 larvae.
Moreover it is worth noticing that CPTIO-treated hemocytes showed adherence to glass and smooth membrane appearance, thereby demonstrating the fitness of the hemocytes under these experimental conditions (Figures 5 and 6).

\section{DISCUSSION}

Nitric oxide (NO) is a highly reactive and unstable free radical gas that is produced by the oxidation of L-arginine to citrulline mediated by the enzyme NO synthase (NOS) (Rivero 2006). The study of NO in insect immunity has been directed primarily to the potentially bactericidal effect of NO and to the signaling pathway triggered upon gut infection in mosquitoes and Drosophila melanogaster (Rivero 2006; Davies and Dow 2009; Hillyer and Estévez-Lao 2010). 

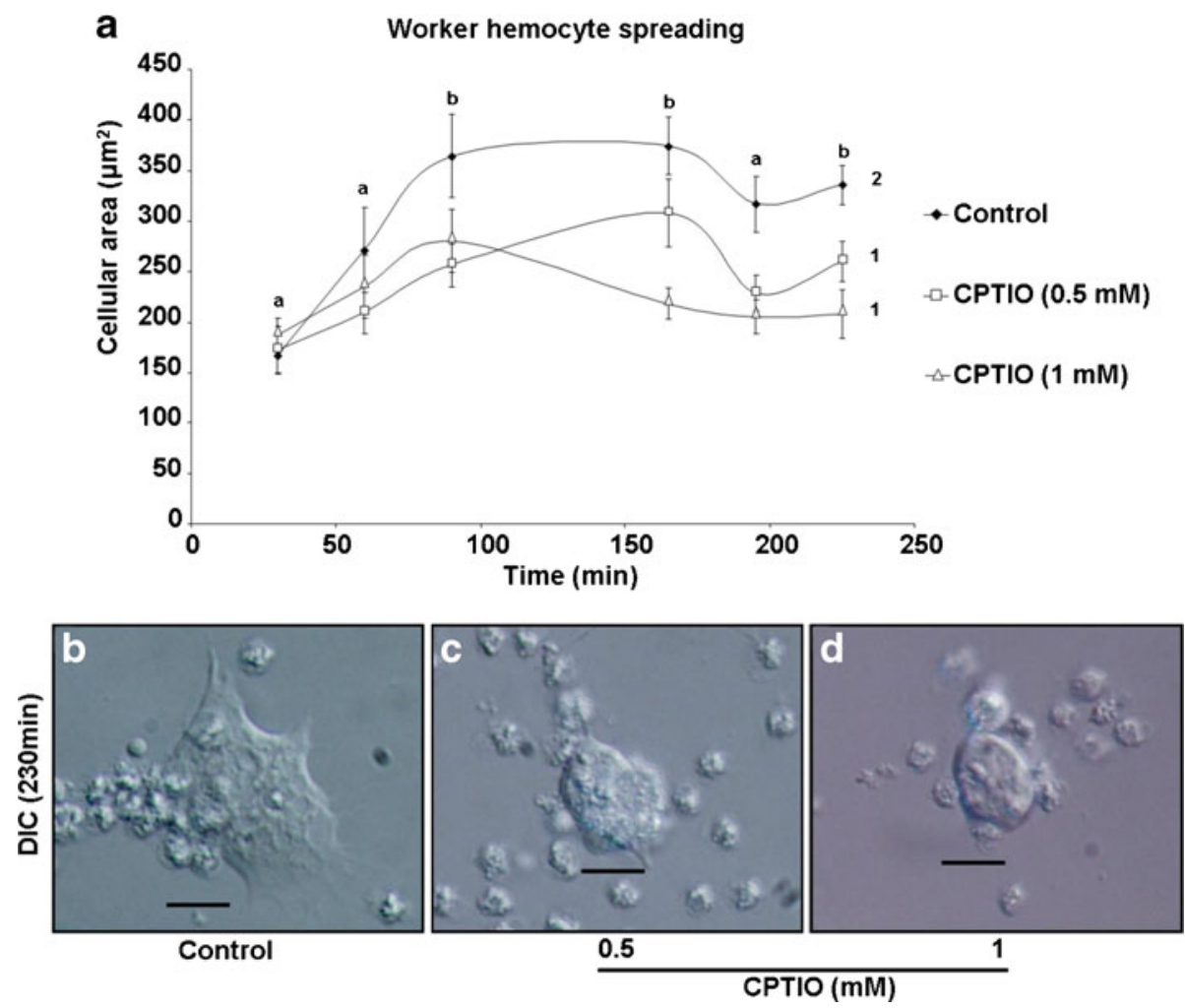

Figure 5. Hemocyte spreading is NO dependent. Time course analysis. Adherent-to-glass worker hemocytes were cultured in 96-well glass-bottom plates. At different time points, pictures were taken and the cellular area was measured using ImageJ software (a). Adherent-to-glass worker hemocytes were cultured in media alone (control, b) or media plus 0.5 or $1 \mathrm{mM}$ of the NO scavenger CPTIO $(0.5 \mathrm{mM}, \mathbf{c} ; 1 \mathrm{mM}, \mathbf{d})$ and observed in an inverted microscope. Hemocyte spreading was analyzed for CPTIO treatments compared to the control group (two-way ANOVA, $f=8.78, P=0.006$ ). Hemocyte spreading through time was analyzed for each treatment (two-way ANOVA, $f=4.98, P=0.01$ ). Different numbers mean significant differences between treatments (Bonferroni correction, $P<0.01$ ). Different letters mean significant differences with respect to $T 30 \mathrm{~min}$ (Bonferroni correction, $P<0.01$ ). Scale bar $=10 \mu \mathrm{m}$.

However, there are no insights about the NO functions as a signal molecule in A. mellifera hemocytes after non-self recognition. Insect hemocyte spreading is considered as the first step of immune activation after recognition of foreign surfaces. This work is one of the first evidences regarding the role of NO in $A$. mellifera immune defense. The experimental evidences support that A. mellifera hemocyte spreading after non-self recognition is dependent on NO production.

The relationship between NO production between $A$. mellifera hemocytes and non-self recognition was established. This was achieved by analyzing the combined results obtained when challenging the hemocytes with abiotic (glass, as general non-self) and biotic non-self. Adherent-to-glass hemocyte type produced large amounts of NO after glass contact compared with the non-adherent-to-glass type. In addition, the nodulation experiments revealed that NO production was strictly restricted to the hemocytes involved in the nodulation response after bacterial challenge. These results suggest that NO could be playing a role after non-self recognition in $A$. mellifera cellular immune response. 

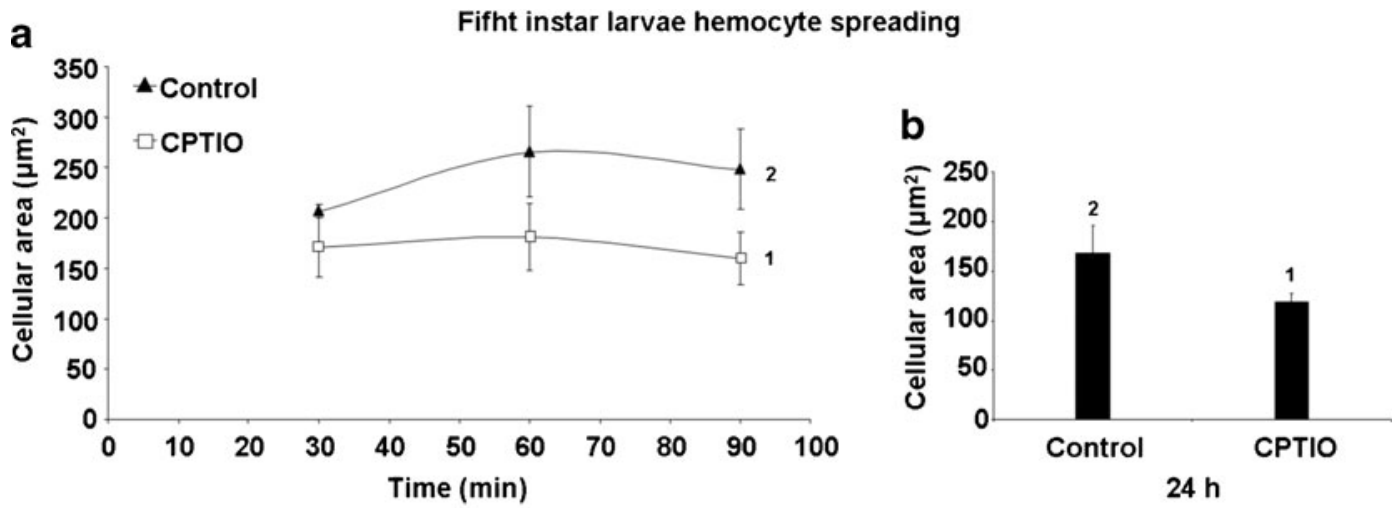

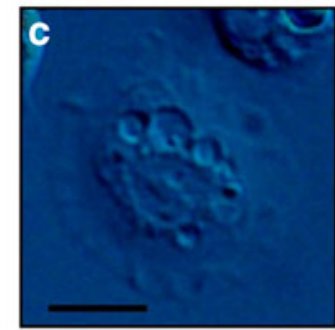

Control

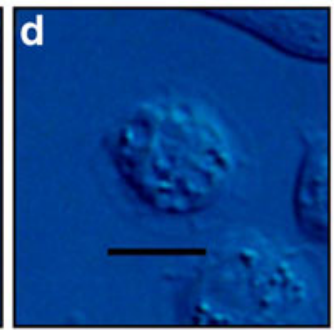

CPTIO

$60 \mathrm{~min}$

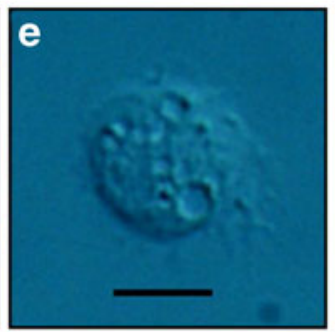

Control

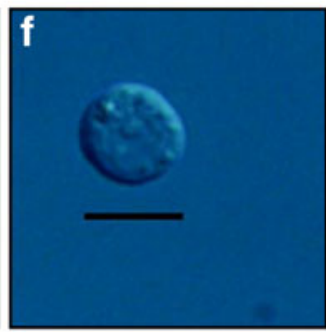

CPTIO

$24 \mathrm{~h}$

Figure 6. Nitric oxide (NO) is required for fifth instar larvae hemocytes spreading upon glass recognition. Adherent-to-glass fifth instar larvae hemocytes were cultured in 96-well glass-bottom plates. At different time points, pictures were taken and the cellular area was measured using ImageJ software (a, b). Adherent-to-glass fifth instar larvae hemocytes were cultured in media alone (control, c, e) or media plus $1 \mathrm{mM}$ of the NO scavenger CPTIO (d, f) and observed by an inverted microscope. Hemocyte spreading was analyzed for CPTIO treatments compared to the control group (two-way ANOVA, $f=24.65, P=0.01$ ). Hemocyte spreading through time for each treatment was analyzed (two-way ANOVA, $f=6.96, P=0.07$ ). Different numbers mean significant differences between treatments. Scale bar $=10 \mu \mathrm{m}$.

This work evidenced that NO could trigger hemocyte spreading per se. The experiments supporting this statement were focused on the hemocyte type that first adheres to glass surfaces. The NO donor SNP and the NO scavenger CPTIO were used to support the $\mathrm{NO}$ involvement in hemocyte spreading. The results indicate that the SNP treatment enhanced hemocyte spreading and that CPTIO reduced the immune activation phenotype. Since hemocyte spreading is a dynamic (non-static) process, the effect of endogenous NO scavenging was also studied in a time course to discard any toxic effects of CPTIO. The fact that CPTIO-treated hemocytes reflected like the control non-treated hemocytes did showed the viability of the CPTIO-treated cells. In addition, NO scavenging reduced hemocyte spreading both in workers and fifth instar larvae. These results highlight the relevance of the functional analysis of A. mellifera hemocytes to study immune responses in vitro. In this case, NO proved to be a crucial molecule to respond to non-self recognition in hemocytes from two different stages that were reported to have different appearance, though behave similarly after glass contact in vitro (Negri et al. 2012b).

The combination of the results discussed above suggests that NO is triggered after nonself recognition and participates in the first steps of immune activation in $A$. mellifera hemocytes. 
However, hemocyte attachment to glass might not be dependent on NO because CPTIO-treated hemocytes remained adherent over time.

Hemocyte spreading over glass surfaces is widely used as a measure of immune aptitude in studies on insect immunity. However, the way in which insects recognize abiotic targets remains a topic of concern. Molecules like calcium or eicosanoids have been reported to participate in hemocyte spreading after non-self recognition (Mandato et al. 1996; Tojo et al. 2000). Here, it is demonstrated that the free radical NO should be considered as a new molecule involved in immune activation of $A$. mellifera hemocytes. The connections between NO and other signal molecules in immune responses could be the platform for future research work in insect immunology.

To go deeper into NO signaling within honeybee hemocytes, NOS gene expression and protein synthesis kinetics should be studied. In vertebrates, two main types of the enzyme have been found: cNOS and iNOS. The cNOS isoform is characterized by the rapid activation (and inactivation) through changes in intracellular calcium levels. In contrast, the activation of the iNOS isoform is $\mathrm{Ca}^{2+}$ independent. To date, only a single NOS isoform has been found in each invertebrate species, with either a constitutive (signaling) or an inducible (toxic) role. In some cases the same isoform has been found to have both roles (Rivero 2006). The presence of a single NOS gene has been reported within A. mellifera genome (AmNOS, AB204558). In addition, NOS activity has been reported in honeybee brain, making NO a key signaling molecule for learning (Müller and Hildebrandt 1995; Müller 1996; Müller and Hildebrandt 2002). This work shows that $A$. mellifera hemocytes produced NO even when cultured in anticoagulant buffer (with the calcium scavenger EDTA). In this sense, the calcium-independent NO production within adherent-to-glass hemocytes suggests the presence of an inducible-like NOS activity in $A$. mellifera. However, more experiments should be performed to support this hypothesis.

Due to its nature NO could either act as a second messenger within each hemocyte or work as a signal between contiguous cells. This work shows that NO is released prior to $A$. mellifera hemocyte immune activation suggesting that NO should be taken into account when studying and understanding the overall cellular immune responses in honeybees.

\section{ACKNOWLEDGMENTS}

This research was supported by "ANPCyT," Argentina (PICT Redes 2006 no. 00890 Nodo 01 and PICT 2011 2383). The authors would like to thank CONICET and the UNMdP. Pedro Negri is a doctoral fellow from CONICET, Argentina. The authors are very grateful to Dr. Claudia Studdert for E. coli strain and to Lic. Leonardo De Feudis for beekeeping support.

L'oxyde nitrique intervient dans les premières étapes de la réponse immunitaire cellulaire d'Apis mellifera à la reconnaissance du non-soi

Apidae/ immunité / oxyde nitrique/ infection

Stickstoffmonoxid spielt eine Rolle in den ersten Schritten der Aktivierung der zellulären Immunantwort bei Apis mellifera

Apis mellifera/ Immunität/ Stickstoffmonoxid

\section{REFERENCES}

Clark, K.D., Pech, L.L., Strand, M.R. (1997) Isolation and Identification of a plasmatocyte-spreading peptide from the hemolymph of the Lepidopteran insect Pseudoplusia includens. J. Biol. Chem. 272, 23440-23447

Davies, S., Dow, J. (2009) Modulation of epithelial innate immunity by autocrine production of nitric oxide. Gen. Comp. Endocrinol. 162, 113-121

Dean, P., Richards, E.H., Edwards, J.P., Reynolds, S.E., Charnley, K. (2004) Microbial infection causes the appearance of hemocytes with extreme spreading ability in monolayers of the tobacco hornworm Manduca sexta. Dev. Comp. Immunol. 28, 689-700

Dietemann, V., Pflugfelder, J., Anderson, D., Charrière, J.D., Chejanovsky, N., et al. (2012) Varroa destructor: research avenues towards sustainable control. J Apic Res 51, 125-132

Eleftherianos, I., Xu, M., Yadi, H., Ffrench-Constant, R.H., Reynolds, S.E. (2009) Plasmatocyte-spreading peptide (PSP) plays a central role in insect cellular immune defences against bacterial infection. J. Exp. Biol. 212, 1840-1848

Hendriksma, H.P., Hartel, S., Steffan-Dewenter, I. (2011) Honey bee risk assessment: new approaches for in vitro larvae rearing and data analyses. Met. Ecol. Evol.. doi:10.1111/j.2041-210X.2011.00099.x 
Hillyer, J., Estévez-Lao, T. (2010) Nitric oxide is an essential component of the hemocyte-mediated mosquito immune response against bacteria. Dev. Comp. Immunol. 34, 141-149

Gillespie, J.P., Kanost, M.R. (1997) Biological mediators of insect immunity. Annu. Rev. Entomol. 42, 611-643

Levy, S. (2012) What's best for bees. Nature 479, 164-165

Ling, E., Yu, X. (2005) Prophenoloxidase binds to the surface of hemocytes and is involved in hemocyte melanization in Manduca sexta. Insect Biochem. Mol. Biol. 35, 1356-1366

Mandato, C.A., Diehl-Jones, W., Moore, S.J., Downer, R.G.H. (1996) The effects of eicosanoid biosynthesis inhibitors on prophenoloxidase activation. phagocytosis and cell spreading in Galleria mellonella. J. Insect Physiol. 43, 1-8

Marmaras, V.J., Lampropoulou, M. (2009) Regulators and signalling in insect haemocyte immunity. Cell. Signal. 21, 186-195

Martin, S.J., Highfield, A.C., Brettell, L., Villalobos, E.M., Budge, G.E., Powell, M., Nikaido, S., Schroeder, D.C. (2012) Global honey bee viral landscape altered by a parasitic mite. Science 336, 1304

Müller, U., Hildebrandt, H. (1995) The nitric oxide cGMP system in the antennal lobe of Apis mellifera is implicated in integrative processing of chemosensory stimuli. Europ. J. Neurosci 7, 2240-2248

Müller, U. (1996) Inhibition of nitric oxide synthase impairs a distinct form of long-term memory in the honeybee, Apis mellifera. Neuron 16, 541-549

Müller, U., Hildebrandt, H. (2002) Nitric oxide/cGMPmediated protein kinase $\mathrm{A}$ activation in the antennal lobes plays an important role in appetitive reflex habituation in the honeybee. J. Neurosci. 22, 8739-8747

Nardi, J.B., Pilas, B., Bee, M.C., Zhuang, S., Garsha, K., Kanost, M.R. (2006) Neuroglian-positive plasmatocytes of Manduca sexta and the initiation of hemocyte attachment to foreign surfaces. Dev. Comp. Immunol. 30, $47-462$
Negri P., Maggi D., Lamattina L., Eguaras M. J. (2012a) Nitric oxide participation in Apis mellifera hemocytic immune activation upon recognition of non-self and encapsulation. 2012 International Congress on Invertebrate Pathology and Microbial Control and 45th Annual Meeting of the Society for Invertebrate Pathology, August 9, Buenos Aires, Argentina.

Negri P., Maggi M. D., Fernandez N., Lamattina L., Eguaras M. J. (2012b) Apis mellifera cellular immune responses in-vitro: qualitative differences before and after the puppae metamorphosis black box. 2012 International Congress on Invertebrate Pathology and Microbial Control and 45th Annual Meeting of the Society for Invertebrate Pathology, August 9, Buenos Aires, Argentina, DBI-06, page 57

Pech, L.L., Strand, M.R. (1995) Encapsulation of foreign targets by hemocytes of the moth Pseudoplusia inchdens (Lepidoptera: Noctuidae) involves an RGD-dependent cell adhesion mechanism. J. Insect Physiol. 41, 481-488

Potts, S.G., Biesmeijer, J.C., Kremen, C., Neumann, P., Schweiger, O., Kunin, W.E. (2010) Global pollinator declines: trends, impacts and drivers. Trends Ecol. Evol. 26, 345-353

Rivero, A. (2006) Nitric oxide: an antiparasitic molecule of invertebrates. Trends Parasitol. 22, 219-225

Rosenkranz, P., Aumeier, P., Ziegelmann, B. (2010) Biology and control of Varroa destructor. J. Invertebr. Pathol. 103, 96-119

Strand, M.R. (2008) The insect cellular immune response. Insect Sci 15, 1-14

Tojo, S., Naganuma, F., Arakawa, K., Yokoo, S. (2000) Involvement of both granular cells and plasmatocytes in phagocytic reactions in the greater wax moth, Galleria mellonella. J. Insect Physiol 46, 1129-1135

Whitten, M.M.A., Tew, I.F., Lee, B.L., Ratcliffe, N.A. (2004) A novel role for an insect apolipoprotein (apolipophorin III) in $\beta$-1,3-glucan pattern recognition and cellular encapsulation reactions. J. Immunol. 172, 2177-2185 